\title{
LA TRANSFORMACIÓN DEL PAISAJE FORESTAL EN EL INTERIOR DE LA CUENCA DEL DUERO Y SUS APROVECHAMIENTOS TRADICIONALES (SIGLOS XVI-XVIII)
}

\author{
José María RAMOS SANTOS \\ I. E. S. Jaime Gil de Biedma (Nava de la Asunción. Segovia)
}

\begin{abstract}
RESUMEN: Durante la Edad Moderna los espacios forestales del interior de la Cuenca del Duero fueron objeto de una intensa intervención humana. Este hecho determinó, en unos casos, la transformación de este paisaje vegetal, con la degradación del monte y la sustitución o la preferencia de unas especies arbóreas frente a otras, y, en otros casos provocó la completa desaparición de los espacios forestales. Ahora bien, esta intervención no contradice el hecho constatado de la permanencia de un notable paisaje vegetal con rasgos propios de un paisaje naturalizado, dada la continuada preocupación por la permanencia de unos espacios arbolados con un ininterrumpido aprovechamiento tradicional de leñas, madera, carbón vegetal y pastos. Así, el monte mediterráneo de la Edad Moderna es, con frecuencia, un monte mixto con pinos, encinas, quejigos, robles, enebros e, incluso, alcornoques.
\end{abstract}

PALABRAS CLAVE: Monte bajo, monte mixto, degradación, ordenación, paisaje natural.

ABSTRACT: In the Modern Age the forest landscape of inland of the Duero Basin was the outcome of human action on the natural environment. This brought on the one hand to the transformation of this vegetational landscape, with the degradation of the forest and the substitution or the prefèrence of some arboreal species for other, and on the other hand the forest space began to disappear completely. Well now, this intervention didn't contratict the verified fact of the permance of a outstanding forest landscape whit typical features of a naturalized landscape, the concern to the fact of their continuity over tree-covered spaces took the exploitation of firewood, wood, charcoal and pastures. So, the Mediterranean forest the Modern Age is of the, a mixed forest, with pines, holm-oaks, including cork oaks.

KEY WORDS: Law forest, mixed forest, degradation, ordening, natural landscape.

$\boldsymbol{R} \boldsymbol{E} \boldsymbol{S} \boldsymbol{U} \boldsymbol{M} \boldsymbol{E}_{\text {: }}$ Pendant 1'Âge Moderne le paysage forestier de 1'interieur du Bassin du Duero fût l'objet d'une forte intervention humaine. Ce fait provoqua, quelques fois, la transformation de ce paysage végétal, avec le dépérissement du bois et le remplacement ou la preferérence de quelques essences arborescentes pour des autres, et, en autre cas provoqua une totale disparition du bois. Or, cette intervention humaine non contradît le fait comprové du maintien d'un paysage végétal notable avec des éléments caractéristiques d'un paysage naturalisé, à cause d'une préoccupation continuelle pour la permanence des espaces boisés avec une utilisation ininterrompue de ses usages traditionnels de bois de feu, bois, charbon de bois et pâtures. De cette manière, la forêt méditerranéenne de l'Âge Moderne était, normalement, une forêt complexe avec un peuplement mélangé: Des pines, des chênes verts, des chênes faginés, des chênes pyrénéens, des genévriers thurifères, et même, des chênes lièges.

MOTS-CLÉS: Taillis, forêt mixte, dépérissement, aménagement, paysage naturel.

RESUMO: Durante a Idade Moderna as florestas do interior da Bacia do Douro sofreram uma intensa intervenção humana. Isto determinou, em alguns casos, a mudança desta paisagem vegetal, com a degradação da floresta e a substituição ou a preferência de umas espécies arbóreas por outras, e, noutros casos provocou o desaparecimento total dos espaços florestais. Mas, esta intervenção não contradiz o fato constatado da permanência de uma notável paisagem vegetal com ca- 
racterísticas próprias duma paisagem naturalizada, pela contínua preocupação para conservar alguns espaços arborizados destinados ao aproveitamento, de modo ininterrupto e tradicional, da lenha, da madeira, do carvão vegetal e das pastagens. Assim, a floresta mediterrânea da Idade Moderna é, freqüentemente, uma floresta mista com pinheiros, azinheiras, carvalho-português o carvalho-cerquinho, carvalhos, zimbros e também sobreiros

PALAVRAS CHAVE: floresta baixa, floresta mista, degradação, ordenação, paisagem natural.

\section{INTRODUCCIÓN.}

Un análisis retrospectivo desde la actualidad hacia el siglo XVI permite constatar los profundos cambios que ha experimentado el paisaje vegetal en la Cuenca del Duero. Estas transformaciones han afectado tanto a la superficie ocupada por las masas forestales, como a su localización, a la estructura de tales masas e incluso a la composición en cuanto a especies vegetales de las mismas. ¿A qué se han debido estas transformaciones?. En primer lugar, al hecho de que cada época histórica ha dado una valoración propia al monte y, por extensión, al conjunto del paisaje vegetal. En segundo lugar, porque la intervención humana ha favorecido una selección de aquellas especies vegetales más interesantes para su economía, para la obtención de frutos, madera, leña o pastos en el monte. En tercer lugar, porque las limitaciones físicas, tanto de clima como del tipo de suelos, han representado un condicionante importante para la extensión de unas u otras especies forestales.

El paisaje vegetal resultante de esta intervención humana da lugar a una gran diversidad de formaciones, en monte alto o bajo: En el primer caso son, preferentemente, pinares de pino albar o piñonero (Pinus pinea $\mathrm{L}$.) y de pino negral o resinero (Pinus pinaster Ait.), mientras que el monte bajo corresponde a encinares o carrascales (Quercus ilex subsp. rotundifolia Lam.), quejigares (Quercus faginea Lam.) o enebrales (Juniperus thurifera L.). La opción por el monte bajo es la más generalizada entre las formaciones de quercíneas, tanto por la ventaja que el corto plazo ofrecía a la población rural para el aprovechamiento de leñas, como por la facilidad que estas especies forestales tienen para rebrotar de cepa y de raíz durante amplios períodos de tiempo. El turno de aprovechamiento de este monte está en relación con el destino preferente dado al mismo (aprovechamiento de leña, carbón vegetal o pastos), lo que condiciona unos turnos cortos de 10 a 15 años o turnos medios de 20 a 25 años.

En función de la ordenación seguida estas formaciones arbóreas y arbustivas tendrán una dinámica progresiva, de estabilización o regresiva, conduciendo en este caso hacia distintas formaciones de matorral de sustitución. 


\section{EL PAISAJE VEGETAL ENTRE LOS SIGLOS XVI Y XVIII: LOS CAMBIOS PRODUCIDOS EN SU DISTRIBUCIÓN.}

Es un tópico en la historiografía del siglo XVI referirse a la deforestación del interior de la Cuenca del Duero y a la repoblación con pinares de una amplia zona al Sur del Duero, fundamentalmente durante la segunda mitad del siglo (BENNASSAR, 1989, 37-40). Sin poner en duda esta deforestación, que hubo de producirse, la tendencia sobre el monte debió conducir, más que a su destrucción, a su deterioro y degradación, reduciéndose el número de pies por superficie y la talla de los mismos. En este sentido, la opción que se toma desde antes del siglo XV por el aprovechamiento de las superficies forestales con un sistema de beneficio en monte bajo no es sino el resultado de una fuerte presión sobre el mismo, que obligó a una reducción de los tiempos de corta, con plazos estimados de 15 a 20 años, e incluso menos.

El monte se destruye en el siglo XVI, pero también se conserva y se amplía. Resulta una contradicción, pero también es la definición que mejor se ajusta a la interacción entre el monte y el ser humano. Así, se puede constatar un caso de aumento de plantíos en Tórtoles de Esgueva (Burgos), población que adquiere entre 1588 y 1592 un total de 2.030 obras (cerca de 1.000 Has.) que fueron destinadas a la plantación de un monte de encinas, denominado Monte Nuevo, por oposición al Monte Viejo o de Arriba ya existente. ${ }^{1}$ El interés de esta actuación desde un punto de vista forestal es doble: Por un lado, representa un caso de extensión de una superficie arbolada en un momento en el que no es lo más frecuente en el conjunto de las llanuras del Duero, y por otro significa la orientación de un espacio de monte hacia el encinar como formación arbórea preferente. De estos encinares se obtienen sustanciosos beneficios económicos: Extracción de leña para fabricar carbón vegetal, utilización de la corteza del tronco y de las raíces para las fábricas de curtidos, aprovechamiento de la bellota (ADBU, 1752a).

Hoy contamos con indicios que, sin ser pruebas, sí ponen de manifiesto una tendencia con cierta impronta espacial y reflejan la existencia en el centro de la Cuenca del Duero, entre los siglos XVI y XVIII, de importantes conjuntos de masas arboladas con una indudable riqueza en especies: El modelo de monte

\footnotetext{
${ }^{1}$ En el reinado de Felipe II, ante las crecientes necesidades monetarias para hacer frente a la política en Europa, se incrementaron las ventas de baldíos, tierras de realengo y tierras de propios. En numerosos casos los propios concejos optaron por la compra de unas tierras baldías que ya venían cultivando los campesinos de las localidades afectadas, para lo que tuvieron que suscribir un censo que representó una pesada carga para las arcas municipales de muchas localidades hasta el siglo XVIII. Un completo análisis del problema de los baldíos puede seguirse en GómEZ MENDOZA, J. (1967).
} 
con varias especies, tres o cuatro, podía ser la norma más que la excepción, si bien la continua intervención humana lleva a una reducción de la diversidad de especies arbóreas en cada monte, pues la tendencia iba dirigida a conservar y favorecer aquellas con un mayor interés en cuanto a la producción de leña, carbón vegetal o fruto. La división de los montes en cortas para su explotación lleva a la uniformización de las especies arbóreas en cada corta, para no alterar los tiempos de corta, pues estos eran distintos en el pino, el quejigo y la encina.

Todavía en el siglo XVIII las fuentes escritas constatan la presencia de montes con una cierta diversidad de especies. Así, en Aranda de Duero (Burgos), según se menciona en el Catastro de Ensenada, los montes de encina son seis, con un total de 1.510 fanegas (Carrañuela, Torremilano, La Calabaza, Monte Hermoso, Costajón y Montecillo), y en todos los casos menos en uno (el llamado Montecillo de 100 fanegas) son montes mixtos con quejigos, encinas, enebros y pinos, lo que incide en la diversidad de los montes de las campiñas del Duero. El Montecillo se trata en estas fechas para convertirlo en monte alto, de forma que entre 1700 y 1751 se ha desbrozado dos veces para hacerlo monte alto. Los pinares, por el contrario, han sufrido un proceso de tala, como el Monte Pinar y el Monte Arandilla de 100 fanegas, que, como resultado de recientes roturaciones, ya no tienen arbolado y se han convertido en eriales (ADBU, 1752b).

Hacia el Oeste de esta zona y en el mismo Valle del Duero, en la localidad de Boecillo (Valladolid), el histórico monte de la Vega de Porres, emplazado sobre un extenso manto de arenas, parece en la actualidad el resultado de la evolución hacia un pinar con encinas desde un monte mixto que, todavía a mediados del siglo XVIII, era un monte más complejo. De hecho para varios momentos entre 1758 y 1765 se ha constatado documentalmente la presencia de quejigos (Quercus faginea L.) y alcornoques (Quercus suber L.), además de pinos (Pinus pinaster Ait.) y encinas (Quercus ilex subsp. rotundifolia Lam.), en un contexto en el que el alcornoque no se podía cortar (ARCHVA, 1758, 308-1). Esta circunstancia habla claramente de un monte maduro, pero también de una explotación diversificada, en la que se incluía la extracción del corcho, para esta época del siglo XVIII. ${ }^{2}$

\footnotetext{
${ }^{2}$ El interés de estos datos para valorar los montes con un carácter menos agresivo por el ser humano o montes primarios viene refrendada por la idea que hace años expuso I. G. SIMMONS (1982) en el sentido de que "los montes naturales están a menudo caracterizados por una diversidad de especies. De ordinario, la actividad humana reduce esta diversidad" (320). En este sentido parece innegable que todavía en el siglo XVIII numerosos montes conservaban en las llanuras del Duero un estado suficientemente natural como para que la diversidad de especies fuese algo perceptible por los habitantes de la zona.
} 
En la población de Peñafiel (Valladolid) el concejo es propietario de ocho montes, entre los que destacan el Monte Alto, fundamentalmente de enebro y quejigo, con 4.075 obradas (1.875 hectáreas), y el Monte de los Carrascales, con quejigos, encinas y pinos (Pinus pinaster Ait.), con otras 3.000 obradas (1.380 hectáreas). Los demás montes son de menor extensión, pero de nuevo constituidos por varias especies arbóreas, desde un monte con pinos, quejigos y encinas como en el Monte Vega de Santa Cecilia de 533 obradas (245 hectáreas) y el Monte Nuevo, hasta el quejigar-enebral en el Monte El Campo de Arriba (150 obradas, esto es, 69 hectáreas), el pinar-encinar en el Monte La Rinconada (90 obradas), o el encinar en el Monte Carrascal (10 obradas) (AHPVA, 1752, 194 y 196).

Para insistir en esta coexistencia de pinos y encinas desde épocas históricas, en la Tierra de Pinares segoviana, ya en las Ordenanzas de 1409 de Carbonero el Mayor se establece una protección absoluta sobre el monte encinar de El Reguenal (ASENJO GONZÁLEZ, Ma ${ }^{\mathrm{a}}$, 1986, 331), lo mismo que ocurre con las Ordenanzas algo más tardías de Cuéllar. Todos estos datos determinan una larga pervivencia histórica del encinar en este espacio genuinamente pinariego.

\section{LAS ORDENANZAS DE MONTES DE 1611. SU ÁREA GEOGRÁ- FICA DE INFLUENCIA.}

Estas Ordenanzas se aprueban en Madrid el 23 de junio de 1611 y afectan a un amplio espacio de $9.500 \mathrm{Km}^{2}$ del interior de la Cuenca del Duero, en un radio de 10 leguas en torno a la ciudad de Valladolid. De esta manera, el espacio afectado, siguiendo los principales caminos que comunicaban a la capital castellana con otras ciudades, se extendía por el Norte hasta Ceinos de Campos y Monzón de Valdepero, por el Este hasta Peñafiel, por el Sur hasta Nava de la Asunción, y por el Oeste hasta Toro. En definitiva, en el área reservada se engloban una serie de importantes áreas arboladas como la de los Montes Torozos, la mayor parte del Cerrato y del Valle Esgueva, una parte importante de la Tierra de Pinares (sexmos de Valcorba y Montemayor, Tierra de Portillo, Tierra de Íscar y Tierra de Olmedo), incluyendo buena parte de la Tierra de Cuéllar, y en el valle del Duero los 120 kilómetros comprendidos entre Peñafiel en el Este y Toro en el Oeste.

La delimitación de este espacio no deja de ser conflictiva, pues entra en colisión con las áreas de influencia de otras importantes ciudades castellanas, como Segovia y Zamora, dejando dentro de las diez leguas además de la ciudad de Palencia, otras poblaciones importantes como Cuéllar, Medina del Campo o Peñafiel. ¿Cuáles son los motivos para reservar esta importante área destinada al abasto de carbón vegetal y madera para la ciudad de Valladolid?. En primer lugar, hay que tener en cuenta las grandes necesidades de estos productos en la 
ciudad que fuera capital de la Corte hasta pocos años antes. En segundo lugar parece indudable la existencia en la comarca de unos montes sumamente deteriorados y con una reducida productividad; $y$ en tercer lugar se pretende favorecer el destino de la leña y carbón hacia la ciudad de Valladolid, frente a otros destinos alternativos (Madrid).

\section{Los antecedentes históricos de las Ordenanzas.}

La alarma generada en las altas instancias del poder con relación al estado de los montes a principios del siglo XVII no hace sin poner de relieve una grave situación de deforestación. Esta destrucción de los montes, había sido una práctica frecuente en la época de expansión de la agricultura castellana durante la segunda mitad del siglo XVI, y se había manifestado bien mediante las intrusiones de los campesinos en los montes públicos para la puesta en cultivo de nuevas tierras, bien mediante una iniciativa de los propios concejos para la extensión de los cultivos, o bien mediante la tala indiscriminada de los árboles para obtener un beneficio inmediato con la venta de la madera, puesto que la madera es una materia prima indispensable en los pueblos y ciudades.

En esta dinámica fueron pocos los que se opusieron a la política roturadora. Entre ellos se encontraba el Concejo de la Mesta, que pretendía el mantenimiento de los pastos, y para esto resultaba imprescindible la conservación de los montes, pues una vez que un monte era roturado a continuación se ponía en cultivo, reduciéndose los pastos para el ganado trashumante. Por esta razón durante los siglos XVI y XVII la Mesta interpuso pleitos contra concejos, agricultores o instituciones eclesiásticas por la roturación y puesta en cultivo de montes y dehesas por cuyas proximidades pasaban las cañadas, cordeles o veredas.

En este contexto se enmarcan los pleitos que entabla este Concejo de ganaderos ante la Real Chancillería de Valladolid, entre los que destacan los siguientes: En 1555 contra dos vecinos de Palenzuela (Palencia) en relación con la ocupación de una dehesa; en 1579 contra los concejos de Nava del Rey, Medina del Campo y Pollos (Valladolid) debido a la roturación del monte Valdemuelles y la Dehesa del Palancar (ARCHVA, 1579, 29-2); en 1584 contra el concejo palentino de Vertabillo a causa de la roturación de un terreno que era dehesa (ARCHVA, 1584, 341-1); en 1586 contra el conde de Villanueva en relación con el destino a labranza de unas tierras de la Dehesa de Villaester, que había reclamado la Mesta como tierras de pasto (ARCHVA, 1586, 858-1); en 1588 contra varios vecinos de Támara (Palencia) a causa de la puesta en cultivo de tierras en la Dehesa de Villafruela (ARCHVA, 1588, 1.141-1); en 1589 con el concejo de Toro y los guardas de los montes de Val de Iniestas, El Pego, Bardales, la Reina por no impedir roturaciones (ARCHVA, 1589, 541-1); en 1607 contra el marqués de Aguilafuente en relación con la roturación de unas tierras en la Dehesa de 
Valverde (Palencia) (ARCHVA, 1607, 1.819-1); en 1617 contra los arrendatarios de la dehesa de Las Gordillas (Ávila), al haber roturado parte de la misma en zonas de pasto (ARCHVA, 1617, 2.163-3); en 1768 contra el concejo de Toro debido a roturaciones efectuadas en zona de pastos (ARCHVA, 1768, 380-7).

El mismo motivo de ampliación de los cultivos está en el punto de partida del enfrentamiento entre un gran propietario y algún concejo: La desaparición del arbolado reduce los pastos, circunstancia que perjudica a los propietarios de ganado lanar, especialmente si en el término municipal también se han reducido los montes. Este es el caso del conflicto que en 1619 enfrentó al señor de Foncastín con el concejo de Medina del Campo, cuando el propietario de la dehesa de Foncastín obtuvo Facultad Real para plantarla de viñas. La oposición del concejo de Medina resultó poco eficaz, pues las sucesivas sentencias dictadas por la Real Chancillería de Valladolid terminarán legitimando las roturaciones, lo que alentaba el proceso y suponía una importante merma económica para el municipio medinense.

Otras veces son concejos que comparten el aprovechamiento de un monte común los que se enfrentan entre sí por los abusos cometidos por uno de los concejos en el monte. Así, en 1605 se falla un pleito entre los concejos burgaleses de Nebreda y Pinilla Trasmonte debido a que el segundo de los concejos había realizado una corta de más de 50.000 encinas en el monte Carrascal, común a ambos municipios. El fallo de la Real Chancillería no sólo condena al concejo infractor al pago de una multa de 1.300 ducados, sino que además aboga por el respeto a las normas para la corta del monte: Condenamos al "dicho lugar de Pinilla no haber podido ni poder de aquí adelante talar el monte del Carrascal en la forma que lo han hecho, sino que la tala y corta que de aqui adelante hicieren sea guardando la forma de las leyes y pragmáticas de estos reinos y ordenanzas hechas a la conservación de los montes..." (ARCHVA, 1603, 1.193-1).

Los primeros años del siglo XVII, con la estancia de la Corte en la ciudad de Valladolid, representaron para esta población un importante crecimiento demográfico, habiéndose estimado su censo para estos años en unas 60.000 personas (GUTIÉRREZ ALONSO, 1982,19). Este importante contingente humano tuvo que incrementar la demanda de todo tipo de productos, entre ellos los procedentes del monte: El abasto de leña y madera ocasionó un ostensible deterioro de los montes cercanos a la ciudad, lo que llevó a incrementar la distancia de las áreas forestales de las que se aprovisionaba la ciudad. Tras la marcha de la Corte a Madrid los signos de la crisis económica se hicieron evidentes en la ciudad y en todo el entorno rural, en consonancia con lo que estaba ocurriendo en el conjunto de Castilla (MARCOS MARTín, 2000, 476-477). 
La publicación de unas Ordenanzas para la conservación y ampliación de los montes a principios del siglo XVII es un hecho importante: Se puede valorar en primer lugar como una alternativa a la crisis de la agricultura, pues el monte generaba unas rentas importantes para los concejos y representaba el empleo de mano de obra en la plantación y en la limpieza del mismo. En segundo lugar deben valorarse como una opción a favor del monte, dada la escasez tanto de leña como de carbón en la zona de influencia de la ciudad de Valladolid, hecho que provocaba el encarecimiento de sus precios al tener que transportar estas materias primas desde largas distancias.

\section{El contenido: Su alcance y sus limitaciones.}

El interés de estas Ordenanzas de 1611 es indudable por cuatro motivos: En primer lugar porque se adelantan en el tiempo a la Instrucción de Montes de Pérez Bustamante (1656) y a la Instrucción de Antonio Contreras (1670), lo que revela su modernidad; en segundo lugar, porque ponen de manifiesto la larga tradición existente en el mundo rural en relación con la conservación del monte; en tercer lugar debido a que sus normas se van a conservar en el tiempo, y al menos 150 años después todavía se aplicarán en las cortas de montes; y finalmente, puesto que estas Ordenanzas inciden claramente en la conservación y aumento de los plantíos, con lo que ponen de relieve una práctica común en la ampliación de los encinares mediante la siembra de bellotas o bien para formar monte o para regenerar los existentes, pues la reproducción de cepa y de raíz tenía unos límites temporales. Esta práctica, por otra parte, es anterior a la fecha de redacción de las Ordenanzas, pues en repetidas ocasiones se hace mención a la experiencia como una prueba de que un determinado método es mejor que otro. Así, cuando se explica el método para sembrar los piñones se deja claro que la siembra debe hacerse "lo más en la superficie de la tierra que ser pudiere, porque el piñón, según se tiene por experiencia, si se planta hondo se ahoga".

\section{a) La presentación.}

La imagen que a través de estas Ordenanzas se puede inferir del paisaje forestal resulta ciertamente desoladora. No en vano hay una clara referencia a la destrucción de los montes, que se achaca "a la mala orden que se ha tenido en el uso y aprovechamiento". Frente a esta pérdida de riqueza forestal la Monarquía considera necesario "hacer que los términos de los montes perdidos...tornen a ser montes perfectos como de antes lo eran". El objetivo parece ambicioso, y en parte ilusorio, pues no cabe pensar que algunos años atrás los montes fueran perfectos, dado que el asalto al monte llevaba varios siglos produciéndose. Ahora bien, esta acepción de monte perfecto en este caso hace referencia a montes productivos, intervenidos por el ser humano, y en este sentido se establece en 
las mismas Ordenanzas que desde el mes de octubre al mes de marzo del año siguiente se rocen todos los carrascos y chaparrales del término, "grandes, pequeños, gruesos, delgados". Una vez hecha esta limpieza del monte debe limitarse la entrada de ganados mayores y menores "por el tiempo que os parecie$r e$ ". Conciliar el aprovechamiento de leña con el de pastos es uno de los grandes dilemas de la ordenación de los montes en la Edad Moderna; en todo caso el crecimiento del arbolado es una garantía para la permanencia de los pastos. No en vano, el interés en que las condiciones para el ganado no se deterioren se aprecia en la prohibición de cortar retamas (Retama sphaerocarpa (L.) Boiss) en los montes.

b) La plantación y la conservación de los montes: La estrategia del monte bajo.

En la Edad Moderna el monte bajo era el método acostumbrado de tratamiento del monte de encinas y quejigos (robles en las fuentes de la época), generalmente en turnos cortos que no superaban los veinte años. En esta época el monte bajo es aquél que se explota en turnos cortos, de tal manera que no hay una distinción entre los montes procedentes de siembra o aquellos otros que brotan de cepa: Todos son montes bajos si sus árboles no alcanzan una cierta altura y grosor. En este sentido se entiende que la siembra sea uno de los métodos elegidos para formar o regenerar un monte: La aspiración no es la de formar un monte alto, sino la de constituir una masa arbolada sobre un terreno desnudo de vegetación, o sobre un monte en el que se ha ejecutado una corta a matarrasa, y es preciso restaurar la vegetación.

La razón que tienen las comunidades rurales para practicar este beneficio en monte bajo no es otra que de esta manera se consiguen beneficios inmediatos, pues la vitalidad de las cepas permite la recuperación del arbolado en un corto plazo de tiempo. En tanto que el tratamiento de una masa forestal en monte alto exige espaciar los aprovechamientos de 50 a 100 años, y además limitan o eliminan muchos de los esquilmos tradicionales (pastos). Pocas veces las comunidades rurales podían hacer una previsión tan larga, cuando las necesidades eran inmediatas.

Estas Ordenanzas de 1611 fijan las condiciones que se han de seguir en la plantación de nuevos árboles, montes y pinares. Así, en primer lugar se debe atender la calidad de la tierra: De esta manera, las "tierras recias de barriales y barrancales" sirven para plantar robledales y encinares, mientras que las "tierras delgadas y de arenisca" no resultan apropiadas para el cultivo, pero sí para plantar pinares. Los olmos (Ulmus minor Mill.), sauces (posiblemente Salix purpurea L.), chopos (Populus nigra L.) "y otros árboles que se ponen de rama $y$ de raiz", se pueden plantar en las riberas, arroyos y otros lugares húmedos. 
Este planteamiento del legislador en cuanto a la diferenciación de las especies plantadas en cada tipo de suelo no debe entenderse de una manera rígida, pero sí ofrece una clara intencionalidad en separar los distintos tipos de masas arbóreas, al formarse pinares por una parte, y encinares y robledales por otra. En este último caso tanto quejigares (Quercus faginea Lam.) como rebollares (Quercus pyrenaica Willd.)

En segundo lugar, se han respetar una serie de normas en cuanto a los tiempos $\mathrm{y}$ formas de plantación de las distintas especies forestales. En cuanto al tiempo de siembra las bellotas de encina y roble (quejigo y rebollo) se han de sembrar entre finales de octubre y diciembre, con la simiente del mismo año, preferentemente la más gruesa y sana, "antes de que esté abellonada". Por el contrario, los piñones de pino albar (Pinus pinea L.) se han de plantar en el mes de marzo o en el de septiembre, en tiempo seco, y se utilizará piñón que "no sea añejo ni sacado al fuego", es decir, el piñón debe ser de la última cosecha, y ha de recogerse una vez que la piña se ha abierto, sin utilizar el fuego para acelerar el proceso de apertura. La necesidad de disponer de semilla en abundancia para realizar la siembra planteaba un problema difícil de resolver en ocasiones, pues obligaba a disponer previamente de árboles reproductores en número suficiente en la zona, lo que no siempre resultaba posible por el deterioro de los montes; además, dado que la cosecha no estaba garantizada, la plantación de un monte podía prolongarse durante varios años. ${ }^{3}$

En cuanto a la forma de siembra para conseguir un monte nuevo el método debe ser el siguiente: Las bellotas de roble y encina se han de plantar de dos en dos, en surcos muy profundos, y cada siembra estará separada de la siguiente por una distancia de dos pies; a unos tres pasos se situará el siguiente surco para la siembra, de tal manera que con la tierra levantada de este surco se cubran las semillas del anterior. Se forma así una superficie con un elevado número de posibles plantas, dado que en cada hectárea se forman hasta 33 surcos, y en cada uno de ellos se ponen 200 semillas, lo que daría un total de unos 6.600 árboles en cada hectárea en el caso de que todos echasen raíz (bien en la primera siembra o en las siguientes). Este es un sistema que garantiza un monte con una alta densidad de pies de encina o roble en los primeros años, pues en las primeras cortas se procedería a una limpieza del monte, dado que la encina, en su crecimiento, necesita espacio y luz para desarrollarse, pues no tolera otras especies competidoras. En todo caso durante un tiempo se ofrecía un importante aprovisionamiento de leña a la población rural de la zona.

\footnotetext{
${ }^{3}$ La preferencia que hay en esta Ordenanza por los pinares de pino albar se justifica por el legislador en el hecho de que el pino albar es "de más fruto y aprovechamiento" que el negral ( $\mathrm{Pi}$ nus pinaster Ait.). En definitiva hay un marcado interés por el fruto frente a la resina.
} 
En el caso de los pinares los piñones se han de sembrar de dos en dos, en surcos poco profundos, "porque el piñón, según se tiene por experiencia, si se planta hondo se ahoga y no nace". La técnica que se practica, denominada "a estaca", requiere un considerable número de personas que, con una bolsa colgada al cinto, y provistas de un palo de dos palmos, terminado en punta, se deben desplegar en fila por todo el término objeto de siembra. Estas personas estarán separadas dos pasos unas de otras, y en un hoyo que cada una ha de hacer con la estaca pondrá uno o dos piñones, que a continuación cubrirá con arena sirviéndose del pie. Así se procederá hasta que todo el término haya sido sembrado.

Una vez plantado un término o podados los árboles de un monte para rejuvenecerlos se debe preservar el monte, limitando la entrada de personas o animales. Así, en los montes de roble y encina y en los pinares, tanto en los antiguos como en los nuevamente plantados, no se podrá arrancar ningún árbol, ni cortar leña, ni sacar retama, durante el tiempo que estuvieren vedados. El objetivo es evitar dañar a las plantas, pues las cortas de cualquier árbol se hacían sin el debido cuidado al resto de los pies, con lo que el daño resultaba inevitable. En estos mismos montes no podrán entrar las personas, ni a pie ni a caballo, pues "andando por ellos se maltratan muchas plantas"; la prohibición resulta tajante en invierno, pues con los hielos las plantas se quiebran fácilmente al ser pisadas. Tampoco se podrá practicar la caza en estos montes, pues en este caso son los perros los que provocan destrozos irreparables en las plantas.

Durante el tiempo en que los montes nuevamente plantados permanecen acotados se realizan visitas periódicas para que si alguna planta no hubiera nacido "se tornare a replantar en sus tiempos". Paralelamente, pasados cinco o seis años después de la siembra, deben realizarse las labores de limpieza en los pinares; estas labores consisten, primero en entresacar algunos pies de cada fila, y a continuación, en los pinos que permanecen, podar las ramas más cercanas al suelo ("dos ruedas de las ramas cercanas al suelo a la redonda del pino"), de forma que los árboles crezcan más deprisa.

La preocupación por la recuperación del paisaje vegetal se extiende a las riberas de los arroyos, donde se deben plantar álamos, olmos y sauces. Las plantas deben ser de buen grosor, separadas 10 pies entre sí, puestas con "estaca y ma$c o$ " en hoyos muy profundos; el período de plantación se extiende desde el mes de diciembre a mediados de febrero. La plantación de estos árboles se encarga tanto a los concejos como a los dueños de las fincas por las que transcurren los arroyos, pero con la salvedad de que en el caso de que estos propietarios no realicen la plantación en el tiempo fijado, deberá encargarse el concejo correspondiente, obteniendo de esta forma la propiedad de los árboles plantados. 
Una vez formados los montes están autorizadas las cortas, que deben realizarse entre el primer día del mes de octubre y finales del mes de marzo del año siguiente, es decir, durante la época más fría del año, cuando la actividad vegetativa de la planta es menor. Cada concejo debe señalar las cortas que se deben hacer cada año, dejándolo por escrito y ante notario; esta recomendación trataba evitar el abuso por parte de las propias autoridades municipales o por los particulares. Para la corta de los robles se establece una periodicidad de corta de diez años y para la encina de 15 o más años. En todo caso resulta un tiempo de corta muy reducido, lo que incide en la necesidad de leña para los hogares, pero representa un indudable deterioro para el arbolado. Este deterioro posiblemente esté en el origen de la recuperación de los montes, pues a pesar de la capacidad de la encina de rebrotar de raíz, esta capacidad no es ilimitada, de tal manera que tras un período de cortas determinado se debe proceder a una siembra de bellotas para recuperar el monte.

Las cortas autorizadas se harán "a ras del suelo y a hita", es decir, por el pie, dejando la raíz para que el árbol pueda rebrotar, y entre los mojones o hitos del monte, sin salirse fuera de los límites. El éxito de esta práctica llevó a que todavía en el siglo XVIII se mantuviera en las poblaciones cercanas a Valladolid, como se comprueba en San Miguel del Pino, donde hay constancia de una solicitud de 1742 para la corta del monte titulado Montecillo, y en la autorización obtenida se recogen las condiciones para que se realice la corta, con la previsión de que al cabo de unos años el monte se recupere, de tal manera que la corta se ha de realizar por tranzones, dejando en cada uno encinas distantes unas de otras cien pasos, de esta forma el número de pies madre que se conserva por hectárea es muy reducido, tan sólo de cuatro. La intención con este método es que las encinas broten de cepa, pero al mismo tiempo las bellotas caídas de las encinas-madre se emplearán en la siembra para favorecer el aumento y la mejora del arbolado:

"Y una y otra (corta) se han de hacer ayta, y en ras del suelo. Dejando en ellas sus atalayas de 100 en 100 pasos para que los pastores y otras personas sepan que es monte nuevamente beneficiado y que por tal lo han de guardar, so las penas contenidas en las mencionadas ordenanzas, conforme se ordena por el capitulo veinte. Que para que el referido Montecillo produzca y tenga leña y abrigo para los ganados, conforme se fueren haciendo sus cortas se coteen y demarquen por nuevamente beneficiado, y por medio de edictos... se publique que en seis años primeros siguientes no entren en él ganados ovejunos, de huelga y labor, y el ganado vacuno, cabras y cerdos en ocho años" (AHPVA, 98-3).

El interés en la buena ordenación de las cortas es máximo, puesto que uno de los aprovechamientos más apreciados en el monte es la fabricación de carbón, 
tanto más cuanto que el abasto de carbón vegetal y leña es el objetivo prioritario de todos los montes situados en un radio de 10 leguas (unos 55 kilómetros) de la ciudad de Valladolid. En este sentido, y dado el deterioro observado en los montes en el momento de la redacción de las Ordenanzas, se establece una rigurosa prohibición de hacer carbón en todos los montes situados en un radio de 8 leguas de la ciudad de Valladolid hasta que se hayan recuperado.

\section{c) La normativa sancionadora: Su casuística.}

En la Edad Moderna las Ordenanzas de las distintas poblaciones habían mantenido un fuerte carácter sancionador para los infractores. Esto es algo que ya vemos en las Ordenanzas de Toro de 1761, que reproducen otras de 1503, en las que se determinan las prohibiciones en el Monte de la Reina, esto es: Cortar y rozar leña de encina, estepas (Cistus laurifolius L.) y cepos para los hornos de cal, teja y ladrillo, cazar con perros o ballestas; descascar o cortar leña para descascar. Las penas para quienes contravienen estas prohibiciones son elevadas, así la persona hallada sacando leña de encina del Monte de la Reina era penada con 300 maravedíes. Este referencia a la jara estepa tiene el valor de poner de manifiesto una situación de degradación en este espacio forestal.

En el mismo sentido, una gran extensión de las Ordenanzas de la ciudad de Valladolid y su distrito está dedicada a la fijación de las penas por el incumplimiento de alguna de las normas establecidas: Así, no respetar los tiempos de corta (10 años para el roble y 15 años para la encina) y la técnica aconsejada (" $a$ ras del suelo y a hita") implica un castigo de 200 maravedíes por cada pie cortado. El dinero obtenido con esta penalización se repartirá de la forma siguiente: La mitad será para el concejo o dueño particular del monte, y de la otra mitad se harán dos partes, una para el denunciante, y la otra para la Hacienda real.

En los montes y pinares nuevamente plantados la entrada de ganado queda severamente limitada: Así, no puede entrar ganado vacuno en los ocho años siguientes a que se haga la planta; los cerdos y ganado de labor no pueden entrar en los ocho años siguientes. La pena impuesta en caso de incumplimiento será el pago del daño causado y la entrega de una cabeza de ganado por cada cincuenta, si el hecho ocurre de día, y una cabeza por cada 25 si es de noche. De esta manera las ovejas no podrán entrar en los seis años siguientes a la siembra de las semillas, y quien incumpla esta condición pagará de multa una cabeza de ganado por cada 15 animales si el hecho ocurrió de noche, y una cabeza por cada 30 animales si ocurrió de día. Además, el dueño del ganado deberá pagar en cualquier caso el valor de los daños ocasionados en el monte; la primera vez, permanecerá 15 días en la cárcel si el hecho ocurrió de noche y 10 días si fue durante el día; la segunda vez en que el pastor incurra en este delito la pena será 
doblada; y a la tercera vez el pastor será desterrado por un año del lugar donde hiciere el daño, a una distancia de 10 leguas alrededor.

Si los montes se venden para la corta de leña no se podrá entrar en los mismos durante el tiempo de duración del contrato, bajo pena de 100 maravedíes por cada planta que se corte, si es de día, y si es de noche 200 maravedíes.

Una vez realizada una corta queda prohibida la entrada de cualquier tipo de ganado en los dos años siguientes a la finalización de la corta; el castigo por el incumplimiento de esta norma se fija en una cabeza de ganado por cada 40 animales si el acto se cometió de día y una cabeza por cada 30 animales si fue de noche. Pasado ese tiempo de dos años podrán acceder al monte algunos animales (ovejas), pero para otros se extiende la prohibición hasta los 10 años (ganado de labor), los ocho años (cabras, vacas y bueyes) y los seis años (bestias de huelga). Contravenir esta norma implica un castigo de 100 maravedíes por cada vaca, si es de día, y si es de noche la pena se duplica.

Las Ordenanzas contemplan la circunstancia de las villas que se han eximido de otras jurisdicciones, habiendo sido dotadas de monte propio para los pastos de sus ganados, han arrancado de raíz los montes, repartiendo las tierras entre los vecinos. El hecho tiene difícil solución, pues las tierras cultivadas en montes públicos han pasado a propiedad de sus titulares; la recuperación de estas tierras para el monte resulta jurídicamente complicada, por lo que el legislador trata de evitar que se repitan estos hechos: "Procederéis contra los que los aren en conformidad a la cédula". En los últimos años del reinado de Felipe II se trató de dar una solución al problema de los bienes públicos (baldíos y ejidos) vendiéndolos a los vecinos o al concejo, pues el objetivo era obtener un beneficio económico inmediato para hacer frente a algunas de las muchas necesidades financieras que tenía la Corona en esa época.

Las penas resultan extraordinariamente elevadas, pues está práctica del carboneo ilegal estaba muy extendida y no sólo provocaba grandes destrozos en el monte, sino que las pérdidas económicas para los propietarios de los montes resultaban considerables, pues imposibilitaba la corta regular de leña. Así, según estas Ordenanzas, los infractores serán castigados con una multa de 5.000 maravedíes la primera vez que sean sorprendidos haciendo carbón, la segunda vez con 10.000 maravedíes y 20 días de cárcel, y la tercera vez serán desterrados del lugar donde hubieran hecho el carbón por un tiempo de un año.

Para la conservación de los montes y pinares se debe elegir en cada concejo a las personas necesarias, conocedoras del terreno y con experiencia en la vigilancia del monte. 


\section{LA DIVERSIDAD DE MONTES EN EL SIGLO XVIII: SU APROVECHAMIENTO Y EL MÉTODO DE EXPLOTACIÓN.}

\section{El estado de los montes.}

La impresión que se transmite a partir de la lectura de la Ordenanza de Montes de Fernando VI, de 12 de diciembre de 1748 (leída en el Ayuntamiento de Valladolid el lunes 13 de enero de 1749), es la de una ruina generalizada en los montes españoles, especialmente "en lo respectivo a la Corte y 30 leguas en contorno, hallándose despoblados, talados y cortados por la mayor parte, de lo que resulta faltar a su preciso abasto la leña y carbón que necesita para subsistir". La realidad debía de ser más compleja, pues dado que los pueblos obtenían unos importantes recursos de sus montes (en muchas ocasiones indispensables para sus vecinos), también se preocupaban por su conservación: El monte abastecía de leña para los hogares y para los hornos de cal, madera para la construcción, hornija para los hornos de pan, pastos para el ganado; constituía un refugio necesario para el ganado en invierno.

Ahora bien, no siempre resultaba sencillo conciliar el binomio conservaciónaprovechamiento, puesto que la conservación de un espacio forestal estaba supeditada a los recursos que de él se extraían, y si estos disminuían podía ocurrir que el monte se abandonase para destinar sus tierras a la agricultura: Después recuperar un área forestal era una tarea complicada, que exigía bastantes años y la población rural no siempre aceptaba el coste que implicaba.

Muchas veces era más práctico apropiarse de la leña necesaria en montes ajenos. Esto parece que es lo que ocurre en el monte de Calzada del Coto, propiedad en su vuelo del Monasterio de San Benito de Sahagún, pero que "se corta todos los años y aún todos los días a manchas y retazos" (AHPLE, 1754, 8.014). Los autores de las cortas debían de ser los vecinos de Calzada que teniendo comunidad de pastos en el citado monte podían entrar en él libremente, y a los monjes de San Benito debía resultarles complicado ejercer la vigilancia de ese espacio, tanto por esta libertad de que disponían los vecinos como por el alejamiento del monasterio del monte de Calzada (a unos cinco kilómetros al Suroeste de Sahagún).

\section{El método de explotación de los montes y los resultados de su apro- vechamiento.}

La información contenida en el Catastro de Ensenada permite una valoración de la diversidad de usos en el aprovechamiento de los montes (leña, carbón, pastos) y la variedad de tiempos empleados en las cortas. De ahí se infiere un diferente estado de conservación de los montes, no estando siempre tan deteriorados como se refiere desde la Corte a través de la Ordenanza de 1748. Así, por 
ejemplo, el monte del despoblado de Torremonte es explotado por los vecinos de Santoyo (Palencia) durante cinco años seguidos, dividiéndose en 40 suertes, y después se deja de cortar durante 12 años seguidos. También el monte de Arriba en Castroverde de Cerrato (Palencia) (1.410 hectáreas) se explota a suertes por los vecinos de las cuatro localidades vecinas (Castroverde, Torre de Esgueva, Fombellida y Villalaco) que tienen comunidad en el monte: En total se saca la elevada suma de 2.374 carros de leña al año. Otro monte de esta población, el Monte Verdugal, se divide en cortas, pero con un turno de tan sólo 11 años. El monte del despoblado de Ventosilla en Gumiel de Mercado (Burgos) se divide por manchas, de tal manera que cada año se obtienen 1.940 carros de leña, lo que da 0,7 carros por hectárea.

En el monte de Mucientes (2.100 hectáreas) se sigue un turno de 12 años, los mismos que en los encinares de Valladolid, situados al Sur de la ciudad (La Plana, Montico, Vasarejos y El Sombrío), pero en este caso la capacidad de producción de las distintas cortas debía ser tan diversa que en el informe emitido en 1763 por el diputado de montes, previamente a la autorización de la corta de ese año, se apunta que "cada una de ellas podrá echar de sí en carbón de 9 a 80 arrobas", es decir, hay una diferencia de 1 a 9 entre la corta más productiva y la menos productiva (Libro de Acuerdos, 27-05-1763). También el concejo de Corcos divide su monte de 505 hectáreas en 12 cortas (42 hectáreas por corta), mientras que el de Quintanilla de Trigueros alarga un año el intervalo, al dividir el monte de 605 hectáreas en 13 cortas (46 hectáreas por corta).

En definitiva, la reducción del número de años parece tener como primera explicación, paradójicamente, el deterioro de los montes: Constituye una paradoja el hecho de que un aprovechamiento en turnos cortos conduzca a una degradación del monte, y dada la reducción de producción de leñas haya que acortar aún más el período de las cortas, con lo que el resultado final no será otro que la destrucción de la cubierta arbórea y arbustiva. En segundo lugar, la reducción del número de años se justifica en razón de la conveniencia de dar satisfacción a las necesidades de los vecinos de la población. Este hecho choca con los deseos de los legisladores de alargar los tiempos de corta para garantizar una recuperación del arbolado: Así, los dos montes propiedad del concejo en Castrillo de Onielo se dividen en tan sólo nueve cortas, aprovechándose una cada año; estas cortas se dividían en suertes por los vecinos, en total 120 suertes por año.

Por el contrario, en otros montes, generalmente privados o propiedad de los monasterios, los turnos se alargan hasta cerca de los 25 años o más. Así, en el extenso Monte del Rey ( 2.430 hectáreas) el turno de corta es de 24 años, de forma que el monte se divide en 24 cortas iguales de 100 hectáreas cada una, aprovechándose una por año, de la que se extraen 150 carros (3.600 carros en el conjunto del monte durante los 24 años) (AHPPA, 1752, Libro 8.676). El mismo 
turno de 24 años se sigue en el monte del despoblado del Rebollar (Valle de Cerrato. Palencia) (282 hectáreas) (AHPPA, 1752, Ibíd.)

Ahora bien, la dinámica forestal a veces es regresiva, lo que lleva a reducir el turno de corta, como se puede comprobar en el caso del Monasterio de la Santa Espina (Valladolid), que a finales del siglo XVI ordenó su monte de 1.500 hectáreas en un turno de 30 años, dividiendo el monte en 22 cortas de encina y 8 de roble (con seguridad quejigos), formándose así cortas de unas 50 hectáreas de media (LÓPEZ GARCÍA, 1984, 662-663). En 1752 la situación ha cambiado hasta tal punto que el monte se ha fragmentado en dos sectores: Dos matorrales en la parte Sur, con 720 iguadas (unas 370 Has.), y tres pedazos de monte de encina y quejigo con un total de 1.783 iguadas ( 900 Has.) en el Norte, divididos en 24 cortas (AHPVA, 1752, Libro 89). En definitiva, durante la primera mitad del siglo XVIII se ha abandonado la explotación de seis cortas (las más alejadas del monasterio), seguramente por degradación de la cubierta arbórea.

En cualquier caso, el rendimiento en leña resultaba similar en el Monte del Rey, con el método de las cortas, y en el Monte de Arriba, explotado con el método de la roza, puesto en el primer caso se obtenían 150 carros por cada 100 hectáreas, mientras que en el Monte de Arriba se obtenían en la misma superficie 168 carros. La diferencia está en que en el Monte del Rey el sistema de cortas permite una progresiva recuperación del monte, mientras que en el Monte de Arriba el deterioro del mismo puede resultar irreversible en su conjunto a lo largo de un tiempo prolongado de explotación con este método.

No cabe la menor duda de que el método de aprovechamiento no resultaba indiferente para la conservación de los montes. De hecho los propios contemporáneos percibían con claridad una relación entre las prácticas de aprovechamiento del monte y su deterioro (o conservación). Veamos un caso: Entre 1739 y 1754 el conde de Castrillo y Orgaz y el convento de San Pelayo de Cerrato (Palencia) mantuvieron un litigio sobre el derecho a pastar los ganados del convento en la dehesa de San Pelayo y Tobilla, propia del conde. El pleito se falla a favor del convento, pero en este caso el verdadero interés del pleito está en el contenido de las dos probanzas, una hecha a petición del conde y otra del convento: En ambas se refieren importantes daños en el arbolado de encina, quejigo y enebro, por lo que deben resultar ciertos.

Ahora bien, la diferencia radica en que en el primer caso los daños se achacan a los pastores y guardas del convento, de lo que resulta una gran pérdida para la fabricación de carbón. Mientras que en la probanza del convento se achaca el deterioro en el arbolado a "arrendarla dicho conde no por partes y tajos como debía para su conservación, sino enteramente la mitad de ella como actualmente la tienen arrendada diferentes vecinos de la villa de Cevico Navero, quienes 
sin arreglarse a la ley ni ordenanza alguna, antes bien, usando de la amplia facultad de todo aprovechamiento que se les concede han pasado a arrancar de raiz y descepar la referida dehesa, esterilizándola de leña para lo sucesivo y privando a los ganados del abrigo necesario en tiempo de invierno y que se conserve la hierba en tiempo de verano" (ARCHVA, 1754, 2.246-1). El problema parece claro, y es el sistema de arrancar de raíz las encinas, lo que provoca una disminución del arbolado e impide la regeneración de éste.

\section{DEBATE Y CONCLUSIÓN.}

Entre los siglos XVI y XVIII el monte es una realidad presente en el espacio físico del interior de la Cuenca del Duero. Es un monte caracterizado por una notable diversidad, tanto en lo referente a las especies forestales y arbustivas que lo componen, como en su estado de desarrollo. Ambas cualidades son el resultado, especialmente, de una intervención humana prolongada en el tiempo (BLANCO CASTRO ET AL., 1997, 27). Intervención que mantiene unos rasgos comunes en el conjunto de la Cuenca, pero en la que también se detectan diferencias que son el origen de la diversidad de los montes: Desde aquellos que están formados exclusivamente por encinas o pinos hasta otros en los que la heterogeneidad de especies es la nota dominante (encinas, alcornoques, quejigos y enebros). Otras veces la diferencia se establece entre los montes con pies de porte arbóreo, frente a los matorrales.

El monte ejerce un fuerte atractivo entre las comunidades rurales pues es el principal aporte de los pastos para el ganado, la leña y el carbón vegetal para los hogares, y la madera para las construcciones. Constituye además una reserva de tierras de cultivo, de tal modo que las comunidades rurales que disponen de extensos montes pueden recurrir a ellos en momentos de expansión agraria para cultivar nuevas tierras. La defensa del monte frente a los extraños se realiza tanto mediante apeos y amojonamiento, como con el recurso a la justicia: El monte se convierte en un instrumento de poder, de forma que la pérdida del mismo o su deterioro representa la ruina inmediata para los campesinos más pobres.

La protección sobre el monte se ejerció tanto desde los concejos como desde el Estado, de tal manera que la publicación de las Ordenanzas de Montes de 1611 pone de manifiesto no sólo el interés de la Monarquía en la conservación y aumento de los plantíos de árboles (interés que tiene como objetivo fundamental el suministro de leña y madera a las ciudades y a las industrias), sino también el conocimiento de unas técnicas selvícolas ciertamente modernas: Así, el recurso a la siembra de semillas de encina, roble o pino, frente a la plantación. La siembra es una técnica más segura y barata que la plantación, además da árboles más vigorosos y de un crecimiento más rápido, lo que es una ventaja para conseguir un aprovechamiento más temprano del monte, pues a partir de 
los 15 ó 20 años ya se alcanzan árboles de un cierto talla para realizar en ellos la corta de leña (MONTOYA OLIVER, 1995, 18-19). Otra técnica consiste en la utilización de semillas procedentes del lugar de repoblación y de la última cosecha; la siembra de las semillas se realiza en surcos, con una alta densidad de semillas por hectáreas, hasta el punto de que se pueden conseguir hasta 6.000 plantas jóvenes, de tal manera que se garantice el éxito de la repoblación, pues el número de semillas que no sobreviven es muy elevado.

El método de beneficio preferido en la Edad Moderna es el monte bajo, que, frecuentemente, se ordena dividiéndolo en cortas de 50 a 100 hectáreas de superficie. El turno de corta es variable, desde 9 hasta 25 años, aunque son más frecuentes turnos que van desde los 9 a 15 años, aspecto que determina un deterioro significativo del monte por la imposibilidad de regenerarse. Las cortas constituyen una unidad invariable, de tal forma que cada año debe cortarse aquella corta señalada, y sólo excepcionalmente se hacen dos cortas en un año. Ahora bien, no siempre se recurre a la división del monte en cortas, de tal forma que otras veces el monte se explota por manchas y tajones, cortándose cada año una. Este método determina la formación de montes con pies de distintas edades mezclados, lo que dificulta un aprovechamiento equilibrado del monte, si bien tiene como ventaja un aprovechamiento ajustado a las necesidades del mercado.

\section{FUENTES}

ADBU. Catastro del Marqués de la Ensenada. Libro de respuestas generales y Memorial del Concejo. Signatura 1.015.

ADBU. Catastro del Marqués de la Ensenada. Libro de respuestas generales. Signatura 2.079.

AhPPA. Catastro del Marqués de la Ensenada. Autos generales de despoblados. Libro 8.676.

AHPVA. Catastro de Ensenada. Libro maestro de seglares. Tomos $1^{\circ}$ y $3^{\circ}$. Signatura 194 y 196.

AHPVA. Catastro del Marqués de la Ensenada. Libro maestro de eclesiásticos. Libro 89.

AHPLE. Catastro de Ensenada. Autos originales y Libro de vecinos. Signatura 8.014.

ArchVa. Pleitos Civiles. Pérez Alonso (F.). Caja 29-2.

ArchVA. Pleitos Civiles. Pérez Alonso (F.). Caja 341-1.

ArchVA. Pleitos Civiles. Pérez Alonso (F.). Caja 858-1.

ArChVA. Pleitos Civiles. Pérez Alonso (F.) Caja 1.141-1.

ARCHVA. Pleitos Civiles. Zarandona y Walls (Olv.). Caja 541-1.

ArchVA. Pleitos Civiles. Pérez Alonso (F.). Caja 1.819-1.

ArchVA. Pleitos Civiles. Pérez Alonso (F.). Caja 2.163-3.

ArChVA. Pleitos Civiles. Pérez Alonso (Olv.). Caja 380-7. 
ArchVA. Pleitos Civiles. Pérez Alonso (Olv.). Caja 308-1.

ArchVA. Pleitos Civiles. Pérez Alonso (F.). Caja 1.193-1.

\section{REFERENCIAS BIBLIOGRÁFICAS}

ASENJO GONZÁLEZ, Ma . J. (1986): Segovia. Laciudad y su tierra a finales de medievo. Segovia. Diputación provincial.

BENNASSAR, B. (1989): Valladolid en el siglo de Oro. Una ciudad de Castilla y su entorno agrario en el siglo XVI. Valladolid. Colección Serie Maior. Editorial Ámbito/ Ayuntamiento de Valladolid; 584 pp.

BLANCO CASTRO, E. ET AL. (1997): Los bosques ibéricos. Una interpretación geobotánica. Barcelona, Editorial Planeta, $572 \mathrm{pp}$.

GÓMEZ MENDOZA, J. (1967): «La venta de baldíos y comunales en el siglo XVI.

Estudio de su proceso en Guadalajara». En Revista de Estudios Geográficos. Número 109. Edita C.S.I.C./ Instituto Juan Sebastián Elcano. Madrid, pp. $499 / 559$.

GUTIÉRREZ ALONSO, A. (1982): «Valladolid en el siglo XVII». En Historia de Valladolid. Tomo IV. Valladolid, Edita Ateneo de Valladolid, pp. 13-108. LÓPEZ GARCÍA, J. M. (1984): «Las economías monásticas ante la crisis del siglo XVII: Fray Hernando de Aedo y la reorganización de la abadía de la Santa Espina». En Congreso de Historia Rural. Siglos XV al XIX. Madrid, Edita Casa de Velázquez/ Universidad Complutense de Madrid, pp. 659/679.

MARCOS MARTÍN, A. (2000): España en los siglos XVI, XVII y XVIII. Economía y sociedad. Barcelona, Editorial Crítica/ Caja Duero, 751 pp.

MONTOYA OLIVER, J. M. (1995): Técnicas de reforestación con encinas, alcornoques y otras especies de Quercus mediterráneos. Hojas Divulgadoras. Número 7-8. Madrid, Edita Ministerio de Agricultura, Pesca y Alimentación, $32 \mathrm{pp}$.

SIMMONS, I. G. (1982): Biogeografia: Natural y cultural. Barcelona, Editorial Omega, 303 pp. 